\title{
Famílias Negras, Desigualdades, Saúde e Saneamento Básico no Brasil
}

\section{Black Families, Inequalities, Health and Sanitation in Brazil}

\section{Las familias negras, las Desigualdades, Salud y Saneamiento en Brasil}

Sônia Beatriz dos Santos ${ }^{1}$

\section{RESUMO}

O presente artigo busca discutir a relação entre os efeitos das desigualdades - raciais/ étnicas, de gênero e socioeconômicas - sobre as condições de vida e saúde das famílias negras, e, por conseguinte, a relação destas circunstâncias com o acesso ao saneamento básico - considerado um direito sanitário - deste segmento populacional no Brasil. $\mathrm{Na}$ primeira parte do artigo, abordamos a questão da desigualdade racial/étnica e sua

1 Possui pós-doutorado em Raça, Gênero e Política Pública pelo Hubert H. Humphrey Institute of Public Affairs, University of Minnesota (2008-2009). É Doutora em Antropologia pela University of Texas at Austin (2008); mestre em Sociologia com concentração em Antropologia pela Universidade Federal do Rio de Janeiro (2000); especialista em Educação a Distância pelo SENAC-RJ (2012). Integrante do colegiado de docentes e pesquisadores do Programa de PósGraduação em Relações Étnico-raciais, CEFET/RJ - Centro Federal de Educação Tecn. Celso Suckow da Fonseca RJ. Especialista em Ciências Humanas e Sociais da UNESCO no Brasil. E professora do curso de Tecnólogo em Gestão Hospitalar da AVM Faculdade Integrada na modalidade a distância. intersecção com as desigualdades de gênero, e socioeconômicas, como um aspecto limitador do acesso a melhores condições de vida e saúde e aos bens e serviços que garantem estes direitos. E na segunda parte tentamos demonstrar a relação entre o acesso precário à infraestrutura de saneamento básico adequada (ou a inexistência deste provimento) e as condições de desigualdades enfrentadas pelas famílias negras, e que trazem como resultados agravos para a saúde das famílias negras pobres, comprometendo sua qualidade de vida. E por fim, concluímos trazendo uma reflexão acerca do SUS - Sistema Único de Saúde frente as questões apresentadas acerca das condições de desigualdades vivenciadas pelas famílias negras pobres no país.

Palavras-chave: Famílias Negras, Desigualdades, Saneamento Básico, Saúde
ABSTRACT
This article discusses the relationship between the effects of inequalities - racial / ethnic, gender and socioeconomic status - on the living conditions and health of black 
families, and therefore the relationship of these conditions with access to basic sanitation - considered one health law - this population segment in Brazil.

The first part of the paper will addresses the issue of racial/ethnic inequalities and its intersection with gender and socioeconomic ones, as an aspect that limiting the access to better living conditions and health, and also to means and services that can guarantee these rights. And in the second part, we try to demonstrate the relationship between poor access to adequate sanitation infrastructure (or the absence of this provision) and the conditions of inequalities faced by black families, and as a result this situation bring problems to the health of underprivileged black families, compromising their quality of life. Finally, we conclude bringing a reflection about the SUS - National Health System in the face of the issues presented regarding the unequal conditions experienced by the poor black families in the country.

Keywords: Black Families, Inequalities, Sanitation, Health

\section{ABSTRACTO}

En este artículo se analiza la relación entre los efectos de las desigualdades - racial / étnico, el género y el nivel socioeconómico - en las condiciones de vida y la salud de las familias negras, y por lo tanto la relación de estas condiciones con acceso a saneamiento básico - considerados una ley de salud este segmento de la población en Brasil. En la primera parte del documento aborda la cuestión de la desigualdad racial / étnico y su intersección con las desigualdades de género y socioeconómicos, como un aspecto de la limitación del acceso a mejores condiciones de vida y la salud y los bienes y servicios que garantizan estos derechos. Y en la segunda parte tratamos de demostrar la relación entre la falta de acceso a la infraestructura de saneamiento adecuada (o la ausencia de esta disposición) y las condiciones de desigualdad que enfrentan las familias negras, y como resultado traen problemas a la salud de las familias pobres negros, comprometiendo su calidad de vida. Por último, llegamos a la conclusión trayendo una reflexión sobre el SUS - Sistema Nacional de Salud frente a los problemas presentados. Por último, llegamos a la conclusión trayendo una reflexión sobre el SUS - Sistema Nacional de Salud adelante las cuestiones planteadas acerca de las condiciones de desigualdad que experimentan las familias negras pobres en el país.

Palabras clave: las familias negras, las desigualdades, saneamiento, salud

\section{INTRODUÇÃO}

O presente artigo busca discutir a relação entre os efeitos das desigualdades - raciais/ étnicas, de gênero e socioeconômicas - sobre as condições de vida e saúde das famílias negras, e, por conseguinte, a relação destas circunstâncias com o acesso ao saneamento básico - considerado um direito sanitário deste segmento populacional no Brasil. Vários estudos têm demonstrado que as desigualdades raciais/étnicas em intersecção com outras formas de iniquidade como a iniquidade como a discriminação de gênero e as desvantagens socioeconômicas acarretam uma maior exposição das famílias negras a situações de vulnerabilidade - individual, social, e 
programática $^{2}$. Um dos efeitos desta intersecção pode ser percebido em termos do acesso a moradia com infraestrutura inapropriada. A exposição de indivíduos a acentuados níveis de desigualdade os têm levado a residir em locais com infraestrutura precária ou inexistente: com sério comprometimento de abastecimento de água potável, de coleta e tratamento de esgoto, limpeza urbana, manejo de resíduos sólidos, manejo de água pluvial que são essenciais para a prevenção de enchentes, controle de pragas, dentre outros fatores. Assim, estamos inferindo que uma das consequências dramáticas da desigualdade racial/étnica no Brasil tem sido o de relegar uma parcela significativa da população brasileira - constituída de pretos e pardos em sua maioria - a conviver em seu cotidiano com uma série de privações devido ao meio ambiente degradado, as quais comprometem sua saúde física e mental, podendo levar a quadros de morbidade e mortalidade graves. $\mathrm{O}$ artigo está dividido em duas partes.

$\mathrm{Na}$ primeira, discorreremos sobre o problema das desigualdades, em específico a desigualdade racial/étnica e sua intersecção com as desigualdades de gênero, e socioeconômicas, como um aspecto limitador do acesso a melhores condições de vida e saúde e aos bens e serviços que garantem estes direitos. E na segunda parte procuraremos demonstrar a relação entre o acesso precário à infraestrutura de saneamento básico adequada (ou a inexistência deste provimento) e as condições de desigualdades enfrentadas pelas famílias negras, e que trazem como resultados

2 (ver Davis, 1981; Bayne-Smith, 1996; Crenshaw, 2000; Sant'Anna, 2000; Loue, 2003; Institute of Medicine of The National Academies, 2003; Werneck, 2003a e 2003b; Willians e Collins, 2003. Schulz e Mullins, 2006; dentre outros). agravos para a saúde das famílias negras pobres, comprometendo sua qualidade de vida. E finalmente, concluímos trazendo uma reflexão acerca do SUS - Sistema Único de Saúde frente as questões apresentadas.

\section{O Problema das Desigualdades: aspectos raciais/étnicos, de gênero, e socioeconômicos}

Muitos estudos têm indicado os efeitos negativos da desigualdade em suas múltiplas dimensões na saúde de uma população, de modo que esta pode ser considerada como um aspecto limitador do acesso à saúde de qualidade e aos bens e serviços que garantem este direito. Desde 1960 as desigualdades no Brasil (e América Latina) assumem proporções alarmantes com efeitos profundos sobre as condições de vida e de saúde das populações (Gianini, 1995). Segundo Rosen (1994) “as desigualdades nas condições de saúde (...) em todo o mundo, se ligam, direta e intimamente, à riqueza e à pobreza”. E neste sentido, “ (...) avanços nas condições de vida, nos serviços de saúde e, consequentemente, redução de doenças e mortes, não têm sido uniformes em todo o mundo, ou mesmo nos países de economia bem desenvolvida (p. 250)". Gianini (1995) argumenta que o pertencimento a uma determinada classe social podese constituir num fator explicativo de situações de morbimortalidade, pois tal pertencimento traz consigo "em última instância, o acesso diferencial à infraestrutura de saúde, à quantidade e qualidade da alimentação, (...) à habitação, etc' (Bronfman 1984 citado por Gianini, 1995: p. 21)”. Gianini também demonstra que condições habitacionais insatisfatórias em termos sanitários e grande exposição a agentes potenciais de doença também explicam e produzem mortalidade e 
morbidade. Assim, não há dúvidas de que a desigualdade num sentido mais amplo é por si só desencadeadora de uma série de privações sociais. Estamos particularmente interessados em refletir sobre os efeitos das desigualdades raciais/étnicas, e sua intersecção com as desigualdades de gênero, e socioeconômicas.

As discriminações raciais/étnicas são variáveis de grande relevância na produção das desigualdades, e seus efeitos sobre a população devem ser compreendidos per si e em intersecção com outras formas de opressão, em especial no que tange ao acesso aos serviços e a implementação de ações e políticas públicas na área da saúde.

Em termos raciais/étnicos, o Censo IBGE de 2010 demonstrou que metade da população brasileira $(50,7 \%)$ é constituída por pretos e pardos, um total de 190.732.694 pessoas. Os maiores contingentes desta população se concentram nas regiões Norte e Nordeste do país, notadamente onde o acesso aos serviços de saúde em geral ainda são os mais precários. No entanto, mesmo nas regiões brasileiras com maiores concentrações de riquezas, encontraremos parcela significativa da população negra vivendo em áreas mais empobrecidas. Segundo Fernanda Lopes (2004)

As vias pelas quais o social e o econômico, o político e o cultural influem sobre a saúde de uma população são múltiplas e diferenciadas, segundo a natureza das condições socioeconômicas, o tipo de população, as noções de saúde, doença e os agravos enfrentados. No caso da população negra, o meio ambiente que exclui e nega o direito natural de pertencimento determina condições especiais de vulnerabilidade. Além da inserção social desqualificada, desvalorizada (vulnerabilidade social) e da invisibilidade de suas necessidades reais nas ações e programas de assistência, promoção de saúde e prevenção de doenças (vulnerabilidade programática), mulheres e homens negros vivem em um constante estado defensivo. Essa necessidade infindável de integrar-se e, ao mesmo tempo, proteger-se dos efeitos adversos da integração, pode provocar comportamentos inadequados, doenças psíquicas, psicossociais e físicas (vulnerabilidade individual). (p. 55)

Deste modo, podemos observar que, as desigualdades na área da saúde estão relacionadas a desvantagens estruturais enfrentadas pela população negra quanto a aspectos socioeconômicos, políticos e culturais na sociedade brasileira, que geram um acúmulo de condições sociais desfavoráveis em termos de acesso a bens materiais e culturais, ao trabalho, a educação e saúde, a condições de habitação dignas, dentre outros; "além disso, expõe esta população, sistematicamente, a vários fatores de risco social que afetam diretamente o desempenho em saúde." (Criola, 2007: p.6).

Marta Silva (2007) argumenta que "quanto mais iniquidade produzir uma sociedade, mais os fatores sociais serão determinantes nas condições de saúde da população. Pensando o SUS do ponto de vista das relações raciais/ étnicas, 'observamos que o esforço para criar um sistema de saúde equânime, universal e integral tem produzido resultados tais como a expansão do acesso à saúde, da cobertura dos serviços e da possibilidade de procedimentos de média e alta complexidade. Porém estes resultados têm impacto diferenciado sobre 
brancos e negros", homens e mulheres, jovens, crianças, adultos e idosos no Brasil, "uma vez que foram mantidas as diferenças de desempenho em saúde destes grupos populacionais", em termos de morbidade e mortalidade (p.6).

No que diz respeito à intersecção entre as desigualdades raciais/étnicas e gênero, nosso foco se deterá sobre a varável chefia de família, pois o número de famílias negras chefiadas por mulheres vêm crescendo (IPEA 2011). Segundo os estudos do IPEA (2008), um dos principais indicadores para se perceberem as transformações e as permanências na igualdade, sobretudo entre homens e mulheres, estão nos dados sobre chefia. Ao longo da última década observa-se a manutenção da tendência de aumento na proporção de famílias chefiadas por mulheres, que passou de 19,7\%, em 1993, a 28,8\%, em 2006. Tal tendência vem sendo acompanhada com atenção por pesquisadoras do tema, posto ser um dado que, por um lado, aponta para contextos de precarização da vida e do trabalho feminino3 e, por outro, revela também sobre processos de (des) empoderamento das mulheres. Tratase, portanto, de um importante indicador de desigualdade de gênero (IPEA 2008: p. 3).

Desvantagens sociais também vão ter reflexos na expectativa de vida da população negra. Em outras palavras "pertencimento a um grupo de cor/raça e ao sexo" têm influências na expectativa de vida dos indivíduos (IPEA 2008: p. 3), o estudo demonstrou que: As mulheres vivem mais e os negros, menos. Em 2006, enquanto 9,3\% das mulheres negras tinham 60 anos ou mais de idade, entre as brancas essa proporção era de 12,5\%. Em 1993 tinha-se 7,3\% e 9,4\%, respectivamente, o que indica que, apesar de a expectativa de vida da população ter aumentado, tendo crescido a proporção de idosos nos diferentes grupos populacionais, as desigualdades entre os grupos permanecem. Esta diferença da expectativa de vida pode ser resultante de uma maior vitimização das mulheres negras em decorrência do sexismo e do racismo, que precarizam seu acesso aos serviços de saúde, habitação, emprego e renda, entre outros. No grupo masculino se percebe tendência similar. Em 1993, os negros com 60 anos ou mais eram $6,5 \%$ deste grupo, tendo passado para $7,8 \% \mathrm{em}$ 2006. No grupo de brancos, no mesmo período essa porcentagem varia de $8,2 \%$ para $10,6 \%$ (IPEA, 2008: p. 3).

As diferenças em termos de expectativa de vida entre brancos e negros, é apontada no estudo como "uma das mais perversas facetas das desigualdades raciais existentes em nosso país, pois as únicas justificativas para essas diferenças residem nas piores condições de vida a que negros e negras são submetidos" (IPEA, 2008: p. 3). Adicionalmente, este estudo demonstrou que o tema de gênero ainda revela muitas contradições posto que "muitos são os desafios marcados pelo sexismo enfrentado pelas mulheres nos bancos escolares e na carreira acadêmica, com atenção especial à situação das mulheres negras, que estão em desvantagem em relação às mulheres brancas e homens brancos em todos os indicadores" ( $p$. 20). O estudo revelou que as "em 2009, 65,5\% de mulheres ocupadas, com 16 anos ou mais, tinham pelo menos 9 anos de estudo, contra apenas 48,7\% das mulheres negras". Deste modo ainda há um grande caminho a percorrer na superação das disparidades que envolvem o gênero, a raça e a educação formal no Brasil. 
Quanto à intersecção entre as desigualdades raciais/étnicas e socioeconômicas, esta realidade poderia ser traduzida numa expressão que tem sido muito utilizada por estudiosos das condições de saúde e vida da população negra no Brasil: 'a pobreza tem cor' (Lopes, 2004; Santos, 2000; Jaccoud e Beghnin, 2002).

Estes estudiosos têm enfatizado que a melhoria da qualidade de vida e saúde necessariamente passa pela compreensão da fragmentação das estruturas sociais ocasionadas por fenômenos econômicos, socioculturais, e políticos, no que diz respeito às dimensões individuais, coletivas, locais ou regionais, e globais. Dentre as várias liberdades negadas a um grande contingente de indivíduos, Lopes (2004) afirma que "por vezes, a ausência de liberdades substantivas relaciona-se diretamente com a pobreza econômica, em alguns casos vinculase à carência de serviços públicos e assistência social e, em outros, a violação da liberdade resulta diretamente de uma negação de liberdades políticas e civis”. (p.7-8). Segundo Lopes (2004), "a pobreza no Brasil tem raça/ cor, sexo e etnia" uma realidade que apesar de ainda levantar controvérsias foi comprovada nos estudos elaborados por Jaccoud e Beghnin (2002) que ao analisarem dados sobre a população brasileira considerada pobre entre os anos de 1992 e 2001 revelaram a existência de profundas desigualdades entre negros e brancos no país: a proporção de negros pobres era duas vezes a proporção daquela encontrada entre os brancos, ou seja, $55,3 \%$ versus $28,9 \%$ em 1992 e 46,8\% versus 22,4\% em 2001" (ver Lopes, 2004: p. 8).

As diferenças raciais no que diz respeito aos anos de estudos representam outro exemplo de vinculação entre as questões raciais/étnicas e as iniquidades socioeconômicas. Como demonstra o estudo do IPEA (2008) sobre as desigualdades raciais ainda são muito acentuadas entre negros e brancos no país.

Os negros e negras estão menos presentes nas escolas, apresentam médias de anos de estudo inferiores e taxas de analfabetismo bastante superiores. As desigualdades se ampliam quanto maior o nível de ensino. (...) o acesso ao ensino médio ainda é bastante restrito em nosso país, mas significativamente mais limitado para a população negra, que, por se encontrar nos estratos de menor renda, é mais cedo pressionada a abandonar os estudos e ingressar no mercado de trabalho (IPEA, 2008: p. 5).

No entanto, o estudo do IPEA (2011), revelou que apesar de no decorrer dos anos terem sido identificados "avanços graduais nos números da educação no país, (...) observase a manutenção das desigualdades que têm, historicamente, limitado o acesso, a progressão e as oportunidades, principalmente, da população negra, de nordestinos e da população rural na educação (IPEA 2011: p. 20-21).

Também o acesso da população negra aos benefícios previdenciários está muito marcado pelas desigualdades, pois estes estão diretamente ligados ao acesso ao mercado de trabalho, no qual historicamente negros e negras sempre tiveram acesso precário, criando uma situação de sobrevivência muito precária para as famílias negras. Segundo Lopes e Werneck (s/d),

Ao analisar os diferentes modelos adotados pelo Estado brasileiro: Santas Casas de 
Misericórdia (a partir de 1582), Caixas de Aposentadorias e Pensões/ CAPs (1923),

Institutos de Aposentadorias e Pensões/ IAPs (1926), Serviço de Assistência Médica

Domiciliar/SAMDU (1949), Instituto Nacional de Previdência Social/INPS (1966), Plano de Pronta Ação/ PPA (1968), Instituto Nacional de Assistência Médica da Previdência Social/ INAMPS (1974), Sistema Nacional de Saúde (1975), Programa de Interiorização de Ações de Saúde e Saneamento/ PIASS (1976), Programa de Desenvolvimento de Sistemas Unificados e Descentralizados de Saúde nos Estados/ SUDS(1987), em todos os sistemas verificamos a persistência de estratégias de exclusão da população negra. Dado que negros e negras eram a minoria entre aqueles que detinham vínculos formais de trabalho e a maioria entre os habitantes das regiões onde o Estado estava mais ausente, por exemplo, os estados da região nordeste. Visto por outro ângulo, a constate mudança de modelos, as diferentes tentativas de se constituir formas adequadas de assistência à saúde da população, mesmo em sua insuficiência, traduzem também sinais da pressão constante que os excluídos fizeram e fazem. Ou seja, nos diferentes momentos, o déficit em relação às necessidades de negras e negros por saúde produziu novas alternativas estatais (p.7).

Por tudo que foi discutido nesta reflexão, vimos que quanto à questão da saúde das famílias negras podemos observar e enfatizar que esta vive uma situação paradoxal, vivendo em condições de saneamento básico precários o que acarreta grandes riscos sociais a saúde, seus indivíduos têm menos acesso aos serviços de saúde, o que de imediato terá grande impacto sobre as taxas de morbidade e mortalidade desta população. Assim, por questões que já discutimos em termos das desigualdades enfrentadas pela população negra que leva seus indivíduos a vivenciar vulnerabilidade individual e social, devido ao fato de o sistema de saúde - SUS não ter conseguido ainda garantir o direito a saúde de modo pleno este gera para o grupo uma vulnerabilidade programática. E em especial no que tange ao direito sanitário, o sistema de saúde brasileiro e os governos em suas três esferas possui um grande débito junto à população negra. Adicionalmente, há uma importante correlação entre "o quadro de saúde dos indivíduos e suas características socioeconômicas"; esta associação nos permite observar que "os baixos níveis de renda afetam a vida dos indivíduos como um todo, expondo a contextos deficientes, desgastantes e geradores de doenças. Além disto, evidenciam as desigualdades no acesso aos serviços de saúde”. (IPEA, 2008: p. 5-6). Assim, a população negra é como aponta o estudo do IPEA dependente do Sistema Único de Saúde, mas este fator não lhe tem assegurado o atendimento pleno de suas necessidades em saúde.

\section{Saneamento Básico e Desigualdades} Raciais/étnicas, Socioeconômicas, e de Gênero: as condições de vida e saúde das famílias negras pobres

Ainda que sejam necessários estudos específicos que investiguem esta realidade, podemos afirmar que grande parte das famílias negras residem em morros, favelas, vilas e conjuntos habitacionais, localidades que historicamente apresentam infraestrutura de saneamento básico precário ou inexistente. Segundo Gomes e Heller (2009) "vilas e favelas são regiões das municipalidades que ao longo 
da história do Brasil não foram priorizadas pelas políticas públicas de saneamento básico (p.1)". Os autores argumentam ainda que,

para estes aglomerados urbanos dirigem-se as pessoas de menor poder aquisitivo que, sem condições de arcar com os custos urbanísticos e na ausência de políticas habitacionais que os assistam, recorrem às áreas menos valorizadas do meio urbano, destituídas de saneamento básico, bem como de outros serviços essenciais, para construir suas moradias (p.1).

E neste sentido, por seu histórico de desigualdades as famílias negras pobres e abaixo da linha da pobreza estão entre este grupo de pessoas indicados por Gomes e Heller (2009). Histórica e tradicionalmente as famílias negras têm em sua maioria vivido nos territórios com infraestrutura de serviços públicos irregular ou inexistente. Em muitos casos estas moram em áreas sem regularização fundiária ${ }^{3} \mathrm{o}$ que torna a situação residencial ainda mais complexa do ponto de vista do fornecimento adequado saneamento básico, pois sem o registro público oficial muitos destes locais encontram-se fora do planejamento de urbanização das prefeituras e seus respectivos estados. Assim, Gomes e Heller (2009) enfatizam que a "inexistência de infraestrutura básica" e a "inadequação quanto à posse dos terrenos" se constituem em características marcantes dos "espaços ocupados pelos pobres nas cidades" (p.1);

3 Regularização fundiária é o processo de intervenção pública, sob os aspectos jurídico, físico e social, que objetiva legalizar a permanência de população moradora de áreas urbanas ocupadas em desconformidade com a lei para fins de habitação, implicando acessoriamente melhorias no ambiente urbano do assentamento, no resgate da cidadania e da qualidade de vida da população beneficiária (ALFONSIN, 1997, p.24, apud GOMES e HELLER,2009: p. 2). observa-se que nas áreas periféricas, regiões" onde se concentram os maiores déficits urbanos de acesso aos serviços de saneamento básico, a falta da posse dos terrenos" ainda se constitui num grande problema muitas famílias (p. 3).

Outro dado revelador da situação vulnerável e desigual das condições de habitação e saneamento básico das famílias negras, diz respeito ao número de domicílios localizados em assentamentos subnormais, que é maior entre este grupo. Segundo o IPEA (2011) proporção entre negros e brancos morando nestas residências se "encontrava estável no período considerado (em torno de $4 \%$, o que corresponde a, aproximadamente, 2 milhões de domicílios)", porém, "é importante registrar que deste total, apenas $33,9 \%$ possuem chefia branca, enquanto que em torno de " $66 \%$ apresentam chefia negra" (p. 31). O estudo revelou também que, enquanto o percentual de domicílios em assentamentos subnormais vem diminuindo para os chefes brancos, vem aumentando em especial para aqueles que apresentam chefia de mulheres negras (aumento de 11 pontos percentuais desde 1995). Os dados em geral indicam, portanto, uma significativa melhoria nas condições de habitalidade dos domicílios ao longo dos últimos 15 anos. Entretanto, no que diz respeito aos assentamentos subnormais, a cada vez mais desigual proporção aponta para uma maior e crescente vulnerabilidade nas condições de habitação das famílias chefiadas principalmente por mulheres negras. (IPEA 2011: p. 31)

Gomes e Heller (2009) apontam para as deficiências do país em apresentar uma política clara no que tange às consequências da "falta de regularização no acesso das 
famílias a infraestrutura sanitária” (p.2). E no cenário das políticas públicas ainda há uma complexidade em torno do caminho para a solução destas questões. Gomes e Heller (2009) demonstravam que os Poderes Legislativo, Executivo e Judiciário apresentavam distintas propostas para esta problemática, enquanto que a "área de saneamento básico permanecia alienada desta discussão (p. 2)."

O Instituto Trata Brasil (2010) realizou um diagnóstico da situação do esgotamento sanitário inadequado e os seus impactos na saúde da população em 81 municípios brasileiros com mais de 300 mil habitantes. Os resultados nos permite vislumbrar o contexto de risco social e os tipos de agravos à saúde a que populações que residem em áreas com saneamento básico precário estão sujeitas. No caso específico do segmento populacional objeto desta reflexão - as famílias negras pobres - ele nos ajuda a perceber a situação de vulnerabilidade em saúde enfrentada por este grupo. Em particular, para esta reflexão, utilizaremos os dados do diagnóstico que se referem a municípios do Estado do Rio de Janeiro. Ao observarmos os dados do diagnóstico sobre as médias das taxas de internação por diarreias para os 10 Municípios com as piores coberturas de Esgoto (2003-2008) descobrimos que cinco destes municípios estão localizados no estado do Rio de Janeiro, onde há uma maior concentração da população negra. São eles respectivamente com as seguintes taxas de internação por diarreia: Belford Roxo (396,3), Nova Iguaçu $(277,0)$, São João de Meriti $(216,0)$, Duque de Caxias $(115,2)$, e São Gonçalo $(113,4)^{4}$."

4 Elaborado com base nos dados do Ministério da Saúde (DataSUS). A taxa média de internações por diarreias representa o total de hospitalizações por 100 mil habitantes. (Instituto Trata Brasil, 2010: p. 15).
(Instituto Trata Brasil, 2010: p. 15).

Dados do IPEA 2011 também nos dão uma visão acerca das circunstância de habitação e saneamento dos domicílios no Brasil. O estudo confirma as disparidades enfrentadas por uma parcela significativa da população no país, revelando os efeitos das desigualdades sociais, de raça e de gênero. Entretanto, nem tudo apresentar um cenário tão desanimador, ainda que haja muitas situações complexas a serem sanadas neste campo, o estudo revelou que houve "um aumento gradual na proporção de domicílios com abastecimento adequado de água" (p.31), e neste sentido "o crescimento mais significativo no acesso a este serviço" sobreveio entre as residências chefiadas por pessoas negras (p. 31), como indicam os dados “em 1995, apenas 76,6\% destes domicílios contavam com abastecimento de água adequado; esta proporção chega a 90,1\% em 2009”(p. 31).

Não obstante, ainda que estas conquistas importante tenham ocorrido em benefício da população negra, o estudo IPEA 2011 enfatiza que o aumento desta cobertura nos domicílios das famílias negras "não se equipara à cobertura do serviço entre a população branca" que apresentou um índice de 94,8\% no ano de 2009 (p. 31). E ainda, mais revelador desta lógica das desigualdades que persiste na sociedade brasileira, é a intersecção dos fatores raciais/ étnicos com os socioeconômicos (ou de classe). $\mathrm{O}$ estudo evidenciou que, a menor cobertura do serviço se dá, (...) em domicílios com trabalhadoras domésticas chefes ou cônjuges de cor/raça negra (88,5\%), enquanto, nas casas chefiadas pelas trabalhadoras brancas, este número chega a $93,3 \%$ (p. 31). 
O estudo do IPEA (2011) demonstrou que o esgotamento sanitário se constitui no serviço que proporciona "a menor cobertura populacional com um percentual de domicílios atendidos de apenas 68,9\% em áreas urbanas"; e no caso das resid6encias rurais esta cobertura é menor ainda, situando-se em torno de 31,6\% em 2009 (p. 31). No que se refere à questão de gênero, o estudo não encontrou altercações expressivas entre os domicílios liderados por mulheres e por homens. Todavia, novamente as desigualdades raciais/étnicas se fazem presentes, pois "as diferenças referentes à raça/ cor e à renda são visíveis” (p. 31), revelando que:

a) Enquanto entre a população branca em geral $77,1 \%$ dos domicílios contam com esgotamento sanitário adequado, apenas $60 \%$ da população negra dispõe do serviço;

b) As disparidades se mantêm quando olhamos para os domicílios chefiados por mulheres brancas e negras. Entre as primeiras, a cobertura é de $78,4 \%$, enquanto, entre as últimas, é de apenas $61,8 \%$. Também aqui o aumento mais significativo na cobertura se deu entre os domicílios com chefes negros (12 e 9 pontos percentuais, respectivamente)."(p. 31).

Em conclusão podemos observar tanto pelas informações apresentadas pelo diagnóstico do Instituto Trata Brasil (2010) quanto pelo estudo IPEA (2011), que os dados demonstram que a questão do fornecimento de serviços de saneamento básicos não podem ser unicamente explicados e compreendidos pela situação de

Pobreza a que um grupo se encontra, visto que os piores indicadores se encontram entre a população negra.

\section{CONSIDERAÇÕES FINAIS}

Em conclusão observamos que formas de discriminação baseadas em fatores raciais/ étnicos, de gênero e de classe operam (histórica e cotidianamente) como limitadores do acesso aos direitos das pessoas - seja no que se refere acesso ao mercado de trabalho, à educação, à moradia, e à saúde - e as expõem aos riscos de discriminação ou tratamento injusto, tendo como consequência imediata a produção de um quadro agudo de desigualdades. Em particular, destacamos as famílias negras como um dos segmentos populacionais brasileiros mais afetados por esta contextos social excludente.

Diante destas circunstâncias de disparidades sociais no país, consideramos que as condições de saneamento básico das famílias negras pobres podem ser consideradas como um bom caso para se pensar o lugar de exclusão e marginalização a que este grupo se encontra na sociedade brasileira. Em outras palavras, ao serem obrigado a conviver com uma infraestrutura precária ou inexistente de saneamento básico no que se refere ao provimento de água potável, aos serviços de coleta e tratamento de esgoto, a limpeza urbana, a manipulação e destino adequado de resíduos sólidos, ao manejo de água pluvial, e ao controle de pragas, estes têm vivenciado uma situação grave de violão de seu direito a saúde.

O Estudo do IPEA (2011) demonstra que entre os anos de 1995 e 2009 houve uma redução tanto da pobreza quanto das desigualdades, mesmo que considerando o problema ainda acentuado no país das diferenças regionais, raciais, e de gênero fortemente presentes na sociedade brasileira. Entretanto, ao deter- 
se mais acuradamente sobre os segmentos populacionais por cor ou raça, o estudo revelou que "conquanto a população negra tenha experimentado aumento relativo da renda média per capita superior ao da população branca, a desigualdade permanece expressiva, pois se parte de patamares muito díspares"; dados de 2009 evidenciavam que os negros possuíam, "em média $55 \%$ da renda percebida pelos brancos, e ainda revelou-se que em 1995, a razão de renda era ainda menor (45\%)" (p. $35)$.

Como revelou o estudo do IPEA (2011) "a pirâmide social, esculpida pelas variáveis renda, sexo e raça, sofreu pequena alteração" (p.35), uma realidade ainda complexa para ser modificada. Ainda assim, é importante compreendermos que os principais esforços e estratégias para alterar o quadro de desigualdades em saúde no país devem tomar como referência o SUS - Sistema Único de Saúde que assegura o direito a saúde de qualquer brasileiro, independentemente, de seu grupo de pertencimento por raça/cor, gênero, e situação socioeconômica. Este é um sistema elaborado pela sociedade civil em conjunto com o Estado brasileiro para garantir "atenção e cuidados, com base no direito universal à saúde e na integralidade das ações, abrangendo a vigilância e promoção da saúde, e recuperação de agravos" (Brasil, 2007: p. 7). Em especial, a simples compreensão e prática de seus princípios ético-políticos, deverias ser suficientes para alterar significativamente as disparidades no sistema de saúde brasileiro. São estes,

- a universalidade do acesso, compreendida como a garantia de acesso aos serviços de saúde para toda a população, em todos os níveis de assistência, sem preconceitos ou privilégios de qualquer espécie;

- a integralidade da atenção, como um conjunto articulado e contínuo de ações e serviços preventivos e curativos, individuais e coletivos, em todos os níveis de complexidade do sistema;

- a equidade, que embasa a promoção da igualdade com base no reconhecimento das desigualdades que atingem grupos e indivíduos, e na implementação de ações estratégicas voltadas para sua superação; e

- a participação social, que estabelece o direito da população de participar das instâncias de gestão do SUS, por meio da gestão participativa, e dos conselhos de saúde, que são as instâncias de controle social. Essa participação social significa a coresponsabilidade entre Estado e sociedade civil na produção da saúde, ou seja, na formulação, na execução, no monitoramento e na avaliação das políticas e programas de saúde (Brasil, 2007: p. 7-10).

No entanto, o estabelecimento do SUS como um sistema que assegura atenção e cuidados em saúde não tem sido suficiente para efetivar o direito da população, em especial da população negra, ainda que este segmento passe a partir de 2009 a contar com mais um reforço ao direito a saúde através da publicação no Diário Oficial da Portaria 992 do Ministério da Saúde, a oficialização da Política Nacional de Saúde Integral da População Negra/ PNSIPN.

Existem ainda grandes limitações no que se refere ao sistema de assistência no país. Serviços como o de saneamento 
básico precisam estar articulados “à ação sistemática e intersetorial do Estado sobre os determinantes sociais de saúde", em outras palavras, "é preciso considerar um conjunto de elementos de ordem econômico-social, cultural", e também relacionados às questões de gênero e raciais/étnicas que têm influência direta ou indireta nas condições de saúde da População (p. 7-10); é somente neste sentido que uma reforma sanitária e um sistema de saúde adequados a toda a população podem se efetivar como uma realidade num país acirrado por profundas desigualdades como o Brasil .

\section{REFERÊNCIAS}

Bayne-Smith, Marcia (editor). Race, Gender, and Health. Sage series on race and ethnic relations: v.15. California: Sage Publications, Inc; 1996.

Brasil. Ministério da Saúde. Secretaria de Gestão Estratégica e Participativa. Departamento de Apoio à Gestão Participativa, Caminhos do direito à saúde no Brasil / Ministério da Saúde, Secretaria de Gestão Estratégica e Participativa, Departamento de Apoio à Gestão Participativa.- Brasília : Editora do Ministério da Saúde; 2007. 24 p. (Série B. Textos Básicos de Saúde)

CRENSHAW, Kimberle. Documento para o Encontro de Especialista em Aspectos da

Discriminação Racial relativos ao Gênero. Estudos Feministas, 171. 1/2002.

Criola. Manual Participação e Controle Social para Equidade em Saúde da População Negra. Rio de Janeiro: Criola; 2007.

Davis, Angela Y. Women, Race and Class.
New York: Random House; 1981.

Gianini, RJ. Desigualdade social e saúde na América Latina/ Reinaldo José Gianini. - São Paulo: ANNABLUME; 1995. - (Selo universidade; 31).

Gomes, UAF, Heller, L. Saneamento Básico em Vilas e Favelas: qual o papel da regularização fundiária? Revista VeraCidade Ano IV - N ${ }^{\circ} 5$ - Outubro de 2009.

Instituto Trata Brasil. Esgotamento sanitário inadequado e impactos na saúde da população: um diagnóstico da situação nos 81 municípios brasileiros com mais de 300 mil habitantes. Instituto Trata Brasil. Novembro 2010. Disponível em: www.tratabrasil.org.br.

IPEA. Retrato das desigualdades de gênero e raça / Instituto de Pesquisa Econômica Aplicada. [et al.] - $4^{\mathrm{a}}$ ed. - Brasília: Ipea, 2011. 39 p. : il.

IPEA. Retrato das desigualdades de gênero e raça. - $3^{\mathrm{a}}$ ed. (Análise preliminar dos dados). Instituto de Pesquisa Econômica Aplicada, Fundo de Desenvolvimento das Nações Unidas para a Mulher, Secretaria Especial de Políticas para as Mulheres; 2008.

Institute of Medicine of The National Academies. Unequal Treatment: Confronting Racial and Ethnic Disparities in Health Care. Washington, DC: National Academies Press; 2003.

Jaccoud LB, Beghin N. Desigualdades Raciais no Brasil: um balanço da intervenção governamental. Brasília:IPEA, 2002.

Loue, Sana. Assessing Race, Ethnicity, and 
Gender in Health. New York, NY: Springer; 2006.

Lopes, F. Experiências desiguais ao nascer, viver, adoecer e morrer: tópicos em saúde da população negra no Brasil. In: Batista, LD, Kalckmann, S, Org. Seminário Saúde da População Negra Estado de São Paulo 2004. São Paulo: Instituto de Saúde; 2005. p. 53-101.

Sant'Anna, Wania. Desigualdades Étnico/ Raciais e de Gênero no Brasil: as revelações possíveis do IDH e do IDG. In: Índice de Desenvolvimento ajustado ao Gênero elaborado por assessores e colaboradores da FASE no âmbito do Projeto "Brasil 2000: Novos marcos para as relações raciais". Rio de Janeiro; setembro de 2000.

Santos, M. Por uma outra globalização: do pensamento único à consciência universal. $4^{\mathrm{a}}$. ed. Rio de Janeiro: Record; 2000.

Schulz, Amy J. \& Mullins, Leith (editors). Gender, race, class, and health: intersectional approaches. San Francisco, CA: Jossey-Bass A Wiley Imprin; 2006.

Werneck, J., Lopes, F. Mulheres Negras: Um Olhar sobre as Lutas Sociais e as Políticas Públicas no Brasil. Jurema Werneck e Fernanda Lopes (org.). Rio de Janeiro: Criola; apoio Fundação Heinrich Böll; s/d.

Werneck, Jurema P. Desigualdade Racial em Números 1: Coletânea de Indicadores das Desigualdades Raciais e de Gênero no Brasil. Ed.: Criola. Apoio: Fundação Heinrich Boll; 2003a.

Werneck, Jurema P. Desigualdade Racial em Números 2: Coletânea de Indicadores das
Desigualdades Raciais e de Gênero no Brasil. Ed.: Criola. Apoio: Fundação Heinrich Boll; 2003b.

Willians, David R. and Collins, Chiquita. "Chap. 22: U.S. Socioeconomic and Racial Differences in Health (391- 431)". In: Race, Ethnicity and Health: A Public Health Reader. LaVeist, Thomas (Ed). San Francisco, CA: Jossey-Bass; 2003.
Artigo apresentado em 26/02/2013

Artigo aprovado em 17/07/2013 Artigo publicado no sistema em: 13/09/2013 